\title{
PLASMA LENSES FOR SLAC FINAL FOCUS TEST FACILITY
}

\author{
D. Betz(1), P. Chen( ${ }^{(2)}$, D. Cline (1), M. Gundersen(3), C. Joshi(1), T. Katsouleas (3), \\ J. Norem ${ }^{(4)}$, S. Rajagopalan(1), J. Rosenzweig(1), J. J. Su${ }^{(1)}$, R. Williams ${ }^{(1)}$
}

(1)UCLA; (2)SLAC; (3)USC: (4)ANI .

\begin{abstract}
A collaborative group of accelerator and plasma physicists and engineers has formed with an interest in exploring the use of plasma lenses to meet the needs of future colliders. Analytic and computational models of plasma lenses are briefly reviewed and several design examples for the SLAC Final Focus Test Beam are presented. The examples include discrete, thick, and adiabatic lenses. A potential plasma source with desirable lens characteristics is presented.
\end{abstract}

\section{INTRODUCTION}

In order to maintain event rates in future high energy colliders, there is a need for new focusing technologies capable of producing ever smaller spot sizes. To this end, a collaborative group of accelerator and plasma physicists and engineers has now formed. The U.S. Plasma I ens Group is interested in exploring the use of plasma lenses to meet the needs of future colliders.

Plasma lenses offer the potential for unparalleled focussing strength, $F / \mathrm{r} \sim 300 \mathrm{MG} / \mathrm{cm}$ (at beam and plasma densities on the order of $10^{17} \mathrm{~cm}^{-3}$ ). Furthermore, adiabatic plasma lenses offer the potential of overcoming the Oide limit ${ }^{1}$ on final spot size which limits all other thin lens devices. Two proof-ofprinciple plasma lens experiments have now been successfully performed at $\mathrm{ANL}^{2}$ and University of Tokyo ${ }^{3}$. However, both of these were in very low density plasmas (e.g., $n_{0} \sim 10^{11}$ $\mathrm{cm}^{-3}-10^{13} \mathrm{~cm}^{-3}$ ), and there is a need to demonstrate the plasma lens in the regime of interest; namely, on a beam such as that at SLAC.

In this poster we develop optimized designs for a plasma lens test at the SLAC Final Focus Test Beam (FFTB), planned for early 1993. We first briefly summarize analytic and computational models for plasma lenses in both discrete and adiabatic lens regimes. We conclude with a description of a candidate plasma source with attractive lens properties.

\section{PlaSma LENS MODELS}

Plasma lenses have been described in detail in Refs. 3-8. The focusing mechanisms in an underdense plasma (density $n_{0}$ < nbeam) is dramatically illustrated by the particle-in-cell simulation shown in Fig. 1.
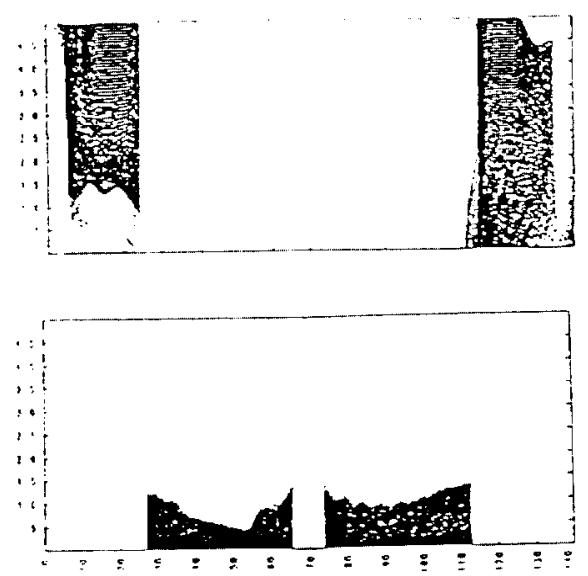

Fig. 1. 2-D PIC simulation of colliding $\mathrm{e}^{-}$and $\mathrm{e}^{+}$beams focussed by plasma lenses. Above: plasma electrons in $\mathrm{r}-\mathrm{z}$ space; Below: $\mathrm{e}^{+}$(right) and $\mathrm{e}^{-}$beams at later time.

The electron beam (left has clearly blown out the plasma electrons in the upper figure, leaving the positive plasma ions (which are massive and move very little on this time scale). This gives a net focusing strength of the lens:

$$
\mathrm{K}=\frac{\mathrm{F}_{\mathrm{r}} / \mathrm{r}}{\gamma \mathrm{mc^{2 }}}=2 \pi \mathrm{n}_{\mathrm{o}} \mathrm{e}^{2} / \gamma \mathrm{mc} \mathrm{c}^{2} \quad\left(\mathrm{n}_{\mathrm{o}}<\mathrm{n}_{\mathrm{b}}\right)
$$

where $n_{O}$ and $n_{b}$ are the plasma and beam density, respectively. We see from the right side of Fig. 1 that the positron beam pulls in the plasma electrons rather than blowing them out. This gives rise to a net focusing strength on the same order.

\section{DESIGN EQUATIONS}

The reduction in the beam beta function $\left(\beta=\sigma^{2} / \varepsilon\right.$, where $\sigma$ is beam spot size and $\varepsilon$ is beam emittance) afforded by a plasma lens is 5

$$
\frac{\beta^{*}}{\beta_{0}^{*}}=\frac{1}{1+K\left(\beta_{0}-\beta_{1}\right) \beta_{0}^{*}} \text {. }
$$

where $K$ is given by Eq. (1), $\beta_{0}^{*}$ is the minimum $\beta$ without the plasma lens and $\beta_{1}$ is the $\beta$-function at the lens exit:

$\beta_{1}=\frac{\beta_{0}}{2}+\frac{1}{2 K \beta_{0}^{*}}+\left(\frac{\beta_{0}}{2}-\frac{1}{2 K \beta_{0}^{*}}\right) \cos v 1+\frac{-2 s_{0}}{v \beta_{o}^{*}} \sin v 1$

$\beta_{O}$ is beta at the lens entrance, $v=2 \sqrt{K}, 1$ is the lens thickness, and $s_{O}$ is the position of the lens entrance relative to the original beam waist (see Fig. 2). 


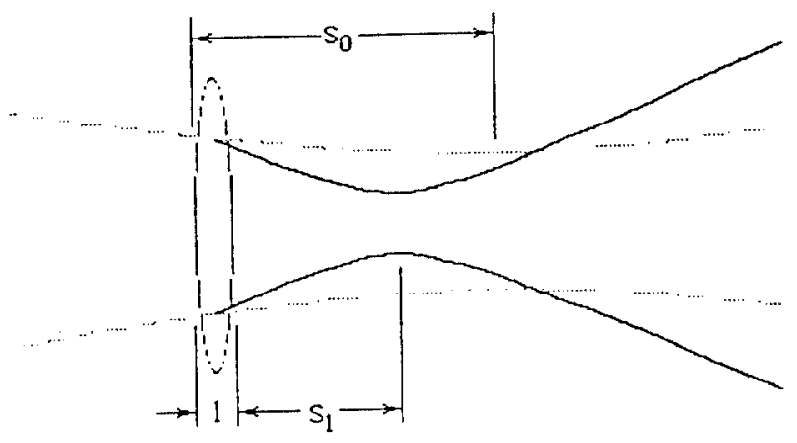

Fig. 2. Illustration of notation used in lens designs.

The position of the lens entrance $s_{0}$ and the distance from the lens exit to the focal point $s_{1}$ are related to beam parameters as follows:

$$
\begin{aligned}
& s_{0}=\sqrt{\beta_{0}^{*}\left(\beta_{0}-\beta_{o}^{*}\right)} \\
& s_{1}=\sqrt{\beta^{*}\left(\beta_{1}-\beta^{*}\right)}
\end{aligned}
$$

\section{ADIABATIC LENS}

Rather than focus a beam to a single focal point, a ramped plasma density $\left(\mathrm{n}_{\mathrm{O}}(\mathrm{s})\right)$ can be used to adiabatically squeeze the equilibrium beam radius ${ }^{7}$. Since there is no single focus, this approach is insensitive to chromatic aberrations that ultimately limit the spot size from all other lenses ${ }^{1}$. For a beam properly matched into a plasma ${ }^{8}$ the $\beta$ - function reduction of the adiabatic lens is

$$
\frac{\beta}{\beta_{0}^{*}}=\left(\frac{n_{0}}{n}\right)^{1 / 2} \frac{1}{1+\alpha^{2}},
$$

where $\mathrm{n}$ is the final plasma density, and the plasma density profile is assumed to be of the form ${ }^{7} \mathrm{n}(\mathrm{s})=\mathrm{n}_{\mathrm{O}}\left(1-2 \alpha \mathrm{s} / \beta_{\mathrm{o}}\right)^{2}$, corresponding to a uniform rate of adiabaticity $(\partial \beta / \partial \mathrm{s}=-2 \alpha=$ constant). Thus the lens length is approximately $1=\beta_{\mathrm{o}} / 2 \alpha$. The matching conditions for the beam and plasma are

$$
\begin{aligned}
& \alpha=s_{\mathrm{o}} / \beta_{\mathrm{o}}^{*}, \\
& \beta_{\mathrm{O}}=\sqrt{\mathrm{K}_{\mathrm{o}}^{-1}\left(1+\alpha^{2}\right)},
\end{aligned}
$$

with $\mathrm{K}_{\mathrm{O}}$ given by (1). A lower limit on $\beta$ (Eq. [6]) arises due to the requirement that $\mathrm{n}_{\mathrm{b}}>\mathrm{n}_{\mathrm{O}}:{ }^{9}$

$$
\beta>\varepsilon_{n} \sqrt{2 \pi} \sigma_{z} / \mathrm{Nr}_{\mathrm{e}}
$$

where $\varepsilon_{n}=\gamma \varepsilon$ is the normalized emittance, $N$ is the number of beam particles, $\mathrm{r}_{\mathrm{e}}$ is the classical electron radius $\mathrm{e}^{2} / \mathrm{mc}^{2}$, and $\sigma_{z}$ is the length of the (assumed Gaussian) bunch.

A preliminary self-consistent PIC simulation of an adiabatic electron lens is shown in Fig. 3. Simulation of adiabatic positron lenses is in progress.

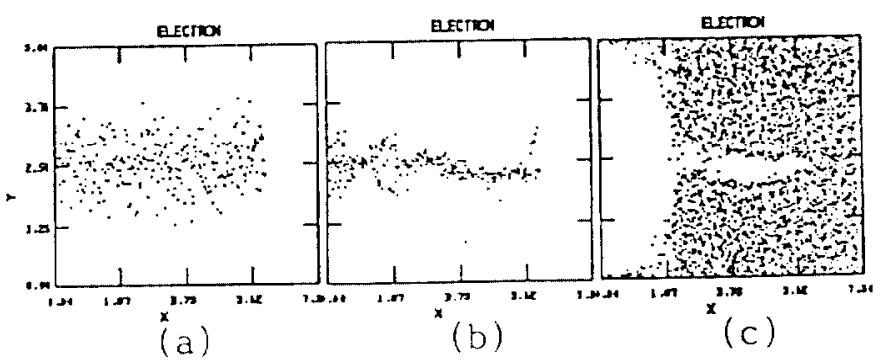

Fig. 3. Adiabatic lens simulation. (a) Plasma electrons showing channel formed by beam; (b) Beam electrons without lens; (c) Beam with lens. $n / n_{O}=1000 ; \alpha=\sqrt{3}$; unmatched beam: $n_{b}(o)=250 n_{0} \exp \left(-2 y^{2} \omega_{p}^{2} / c^{2}\right), \varepsilon=o$.

\section{FFTB DESIGN EXAMPLES}

The design equations (1) - (5) are applied to the FFTB parameters in the table below. The example in the first column is matched to the plasma source described in the next section; for $\varepsilon=3 \times 10^{-8} \mathrm{~cm}$-rad it shows that the final beam size can be reduced from $4.1 \mu$ to $1.9 \mu$ by a $2 \mathrm{~cm}$ plasma of density $6 \times 10^{15} \mathrm{~cm}^{-3}$ inserted $6 \mathrm{~cm}$ before the beam's natural waist. By raising the plasma density by a factor of 3 , the same beam could be focuscd to less than $1 \mu$. The second column example is a thick lens design (the focus is in the plasma) and gives a final spot size of $.44 \mu$; the third is an adiabatic lens; the fourth is a flat beam example. Neglecting synchrotron radiation, the flat beam example focuses to $30 \mathrm{~nm}$ which is below the Oide limit ${ }^{1}$ of $39 \mathrm{~nm}$ for this case. Here we took $\mathrm{n}_{\mathrm{o}} \sim 2 \mathrm{n}_{\mathrm{b}}$ and approximated $\mathrm{K}_{\mathrm{y}} \simeq \mathrm{K}\left(\sigma_{\mathrm{xo}} / \sigma_{\mathrm{yo}_{\mathrm{o}}}\right)$, even though $\mathrm{K}_{\mathrm{y}}$ varies as the beam pinches and distorts.

\section{Plasma SOURCES}

M. Gundersen has developed a plasma source which has several features which are optimal for plasma lens experiments. These include (a) an axial opening to facilitate entry and exit of the beam, (b) vacuum compatible components that will not poison the FFTB vacuum system, (c) precision timing and reproducability, (d) variable plasma density and thickness. This device has been built and operated at USC in the parameter regime described above. In order to implement this system as a lens in the $4 \mathrm{~cm}$ beam pipe of the FFTB, it is necessary to modify the gas flow system and baffles. This design, illustrated in Fig. 4, also minimizes the 
gas flow into the FFTB vacuum system. It appears to be possible (based on recent experiments at U. of Erlangen, W. Germany) to modify the source to reach pulsed densities exceeding $10^{18} \mathrm{~cm}^{-3}$ and ramped density profiles appropriate for an adiabatic plasma lens.

For very dense beams, plasmas produced by beam ionization of a gas are also of interest. Collisional ionization is fairly weak, yielding an ionized density of the order ${ }^{10}$.

$$
n_{\text {ion }} \sim 10^{-3}\left(\frac{\mathrm{P}}{1 \text { Torr }}\right)\left(\frac{\sigma_{\mathrm{Z}}}{1 \mathrm{~mm}}\right) \mathrm{n}_{\text {beam }}
$$

Tunneling ionization becomes significant for beams an order of magnitude denser than FFTB; this topic is currently under investigation. Recently a number of laser experiments have demonstrated laser-ionized plasmas in the $10^{16}-10^{18} \mathrm{~cm}^{-3}$ density regime. ${ }^{11}$

\section{Table 1. Plasma Lens Designs for Parameters Similar to FFTB}

\begin{tabular}{|c|c|c|c|c|}
\hline & $\frac{\text { Discrete }}{\text { Lens }}$ & $\begin{array}{l}\text { Thick } \\
\text { Lens }\end{array}$ & 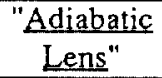 & $\underset{\text { Beam }}{\text { Elat }}$ \\
\hline \multicolumn{5}{|l|}{$\begin{array}{c}\text { Initial } \\
\text { Beam } \\
\text { Parameters } \\
\end{array}$} \\
\hline $\begin{array}{l}\beta_{\mathrm{o}}^{*} \\
(\mathrm{~cm})\end{array}$ & 6 & .75 & 6 & $\begin{array}{l}\beta_{o x}^{*}=.33 \\
\beta_{o y}^{*}=.012\end{array}$ \\
\hline$N$ & $10^{10}$ & $2 \times 10^{10}$ & $10^{10}$ & $2 \times 10^{10}$ \\
\hline$\sigma_{z}$ & $1 \mathrm{~mm}$ & $.5 \mathrm{~mm}$ & $1 \mathrm{~mm}$ & $.5 \mathrm{~mm}$ \\
\hline$\gamma$ & $10^{5}$ & $10^{5}$ & $10^{5}$ & $10^{5}$ \\
\hline$\varepsilon(\mathrm{cm}-\mathrm{rad})$ & $<9 \times 10^{-8}$ & $3 \times 10^{-8}$ & $<12 \times 10^{-8}$ & $\begin{array}{l}3 \times 10^{-8} \\
3 \times 10^{-9}\end{array}$ \\
\hline$\beta_{o}$ & $12 \mathrm{~cm}$ & $.75 \mathrm{~cm}$ & $12 \mathrm{~cm}$ & $\begin{array}{l}4.7 \mathrm{~cm} \\
120 \mathrm{~cm}\end{array}$ \\
\hline \multicolumn{5}{|l|}{$\begin{array}{l}\text { Lens } \\
\text { Parameters } \\
\end{array}$} \\
\hline $\begin{array}{l}\text { Position } \\
\left(\mathrm{s}_{\mathrm{o}}\right) \\
\end{array}$ & $6 \mathrm{~cm}$ & 0 & $6 \mathrm{~cm}$ & $1.2 \mathrm{~cm}$ \\
\hline $\begin{array}{l}\text { Thickness } \\
\text { (1) }\end{array}$ & $2 \mathrm{~cm}$ & $.35 \mathrm{~cm}$ & $\begin{array}{l}3 \mathrm{~cm} \\
(\alpha-1)\end{array}$ & $.5 \mathrm{~cm}$ \\
\hline $\begin{array}{l}\text { Density } \\
\left(\mathrm{cm}^{-3}\right)\end{array}$ & $5.8 \times 10^{15}$ & $1.1 \times 10^{17}$ & $\begin{array}{c}7.8 \times 10^{14} \\
\rightarrow \\
7.8 \times 10^{18} \\
\end{array}$ & $2 \times 10^{17}$ \\
\hline $\begin{array}{l}\text { Focal } \\
\text { distance } \\
\text { from lens } \\
\text { entrance } \\
\left(s_{1}+1\right) \\
\end{array}$ & $4.2 \mathrm{~cm}$ & $.35 \mathrm{~cm}$ & $3 \mathrm{~cm}$ & $.71 \mathrm{~cm}$ \\
\hline$\beta^{*}$ & $1.2 \mathrm{~cm}$ & $.067 \mathrm{~cm}$ & $.24 \mathrm{~cm}$ & $\begin{array}{l}.053 \mathrm{~cm} \\
.0031 \mathrm{~cm}\end{array}$ \\
\hline$\sigma^{*}$ & $1.9 \mu$ & $.44 \mu$ & $.85 \mu$ & $\begin{array}{c}.5 \mu \\
30 \mathrm{~nm}\end{array}$ \\
\hline
\end{tabular}

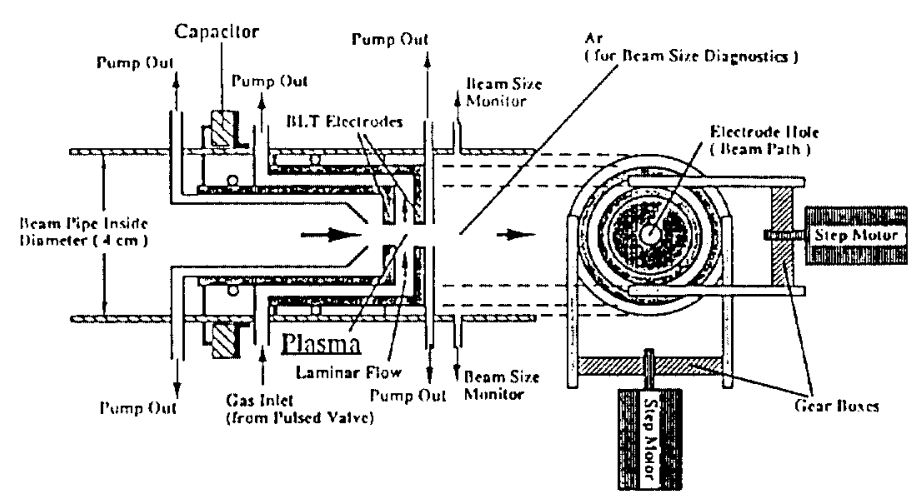

Fig. 4. Proposed structure for BLT plasma lens source.

\section{ACKNOWLEDGEMENTS}

Work supported in part by DOE, NSF, PPRI at LLNL, and ONR N-0014-90-J-1952

\section{REFERENCES}

[1] Oide, K., Phys. Rev. Lett. 61, 1713 (1988).

[2] Rosenzweig, J., et al., Physica Scripta T30, 110 (1990).

[3] Nakanishi, H., et al. Phys. Rev. Lett. 66, 1870 (1991).

[4] Chen, P., Part. Acel. 20, 171 (1987).

[5] Rosenzweig, J. B. and Chen, P., Phys. Rev. D 39, 2039 (1989).

[6] Su, J.J., Katsouleas, T., Dawson, J. M., and Fedele, R. Phys. Rev. A 41, 3321 (1990); Katsoules, T., Su, J.J. Dawson, J. M., Phys. Fluids B 2, 1384 (1990).

[7] Chen, P., Oide, K. Sessler, A. M., and Yu, S.S., Phys. Rev. Lett. 64, 1231 (1990)

[8] Katsouleas, T., and Williams, R. L., Proc. U.S./Japan Workshop on Non-linear Beam Dynamics and Accelerator Mechanisms, Tsukuba, Japan, Oct. 21-24, 1990.

[9] Whittum, D., U.C. Berkeley, Ph.D. Thesis (1990).

[10] Holliday, D., Introductory Nuclear Physics. p.131. John Wiley \& Sons, NY. (1950).

[11] See W. Leemans, et al. and references therein in these Proceedings (Proc. 1991 IEEE PAC, San Francisco, May 7-11, 1991). 